\title{
Interleukin-34 induces pulmonary inflammation in a murine model of lipopolysaccharide-induced acute lung injury
}

\author{
Takaya Ebato*, Shin Ohta, Munehiro Yamaguchi, \\ Hatsuko Mikuni, Hitoshi Ikeda, Megumi Jinno, \\ Kuniaki Hirai, Yoshito Miyata, Hideki Inoue, \\ Tetsuya Homma, Mayumi Yamamoto, Shintaro Suzuki, \\ Akihiko Tanaka and Hironori Sagara
}

Received: 10 May 2021 / Accepted : 8 July 2021

\begin{abstract}
The cytokine interleukin-34 (IL-34) was recently described. However, its role in the lungs is not well understood. IL-34 binds to the colony stimulating factor-1 receptor, thereby enhancing tissue macrophage maturation and differentiation. Macrophages are essential to the airway inflammation process and acute lung injury (ALI). This study aimed to evaluate the role of IL-34 in ALI establishment. C57BL/6 male mice were stimulated intratracheally with lipopolysaccharide (LPS) and sacrificed on day 1, 3, 5, or 7. Additionally, the mice were treated with an anti-IL-34 antibody intranasally before LPS stimulation. The bronchoalveolar lavage fluid (BALF) and lung tissues were collected. The cells of the human peripheral blood monocyte cell line THP-1 and the human airway epithelial cell line BEAS-2B were cultured with LPS in vitro. The total cell number in BALF was higher in the LPS-stimulated mice than in the control mice. The BALF IL-34 level was significantly elevated in BALF on days 1 and 3. IL-34 expression was detected in the pulmonary epithelium in the LPS-stimulated mice on day 1. Anti-IL-34 antibody suppressed the number of macrophages in BALF. IL-34 blockade resulted in pulmonary fibrosis reduction in LPS-stimulated mice on day 5 . LPS stimulation in vitro induced the production of tumor necrosis factor- $a$ (TNF- $a$ ) in THP-1 cells. Furthermore, TNF- $a$ stimulation induced the IL-34 production in BEAS-2B cells. These results suggest that IL-34 induction in the epithelial cells may enhance pulmonary inflammation and fibrosis in the murine model of LPS-induced acute lung injury.
\end{abstract}

Key words :IL-34, LPS, mouse model, acute lung injury, pulmonary fibrosis

\section{Introduction}

Interleukin-34 (IL-34) is a newly described cytokine, which consists of 222 amino acids. Its amino acid sequence is not homologous to other cytokines ${ }^{1}$. In humans, IL-34 mRNA is widely expressed in numerous tissues including the heart, brain, lung, liver, kidney, spleen, thymus, testis, ovary, small intestine, prostate, and colon. The IL-34 expression has been

\footnotetext{
* Corresponding author

凶 Takaya Ebato

doraimu2255@gmail.com

Department of Medicine, Division of Respiratory Medicine and Allergology, Showa University School of Medicine, 1-5-8 Hatanodai, Shinagawa-ku, Tokyo 142-8666, Japan.
}

detected in keratinocytes, epidermis, and neurons ${ }^{1,2}$.

IL-34 shares a common receptor with the macrophage-colony stimulating factor ${ }^{3}$, known as colony stimulating factor-1 receptor (CSF-1R or CD115). IL-34 leads to the formation of inflammatory lesions and autoimmunity because the binding of IL-34 to CSF-1R stimulates the differentiation and proliferation of monocytes and cytokine production, ${ }^{4,5}$.

CSF-1R, a member of the platelet-derived growth factor receptor subfamily, is a transmembrane homodimeric type III receptor encoded by the c-Fms proto-oncogene ${ }^{6}$ that stimulates a tyrosine kinase signaling pathway. The CSF-1R expression is restricted primarily to cells of the mononuclear phagocytic lineage, including macrophage precursors in the bone marrow, monocytes, osteoclasts, and 
tissue macrophages such as liver Kupffer cells and microglia ${ }^{7}$. Moreover, it can also be detected on the surface of some tissue fibroblasts ${ }^{8}$.

The binding of IL-34 to CSF-1R is critical for better survival and differentiation of monocytes, macrophages, and Langerhans cells ${ }^{9}$. The IL-34 production is correlated with autoimmune diseases and chronic inflammatory diseases (e.g., rheumatoid arthritis, Sjogren's syndrome, and gastrointestinal mucosa in inflammatory bowel disease ${ }^{10-12}$.

Acute lung injury (ALI) is caused by direct pulmonary insult and indirect systemic inflammatory responses to severe pneumonia, sepsis, trauma, and major surgery ${ }^{13}$. The lung is one of the most important target organs for proinflammatory mediators secreted during pulmonary infection, sepsis, and trauma ${ }^{14,15}$. While sepsis and trauma can lead to ALI, pulmonary infection and diffuse lung injury can cause systemic inflammatory response syndrome, sepsis, and even septic shock ${ }^{15}$. These clinical syndromes result in significant morbidity and mortality in intensive care units and underscore the interplay between pulmonary and systemic inflammation in promoting disease progression. The study of the pathological ALI mechanisms using the lipopolysaccharide (LPS)-induced murine ALI model is essential, in seeking an effective ALI therapeutic approach. Corticosteroids are commonly used for the treatment of ALI/acute respiratory distress syndrome (ARDS), but do not improve patients' survival rates $^{16}$.

LPS is a major component of gram-negative bacteria, which contributes to the pathogenesis of bacterial infections ${ }^{17}$. LPS-induced animal models provide means to explore the mechanisms of multiple diseases and provide useful information about the discovery of novel biomarkers and drug targets. Therefore, LPS has been previously used to induce murine ALI models.

Monocytes are circulating blood leukocytes that play an important role in tissue homeostasis and inflammatory response, which are essential to innate immunity and combating pathogens ${ }^{18}$. They are unique among peripheral blood cells because they can migrate to various tissues where they differentiate into morphological and functionally heterogeneous cells, including macrophages, myeloid dendritic cells, and osteoclasts, depending on the stimulus ${ }^{19}$. The differentiation of peripheral blood monocytes into resident tissue macrophages can be recapitulated ex vivo by incubation in CSF-1 presence ${ }^{20}$.

This study aimed to investigate the role of IL-34 in pulmonary inflammation and fibrosis in the murine model of LPS-induced ALI.

\section{Materials and methods}

\section{Animals}

C57BL/6 mice (male; age, 8-12 weeks; weight, 20-26 g) were obtained from Saitama Experimental Animals Supply Co. Ltd (Saitama, Japan). The present study and the repeated experiments used 168 mice. All animal experiments were performed after obtaining approval from the Animal Care and Use Committee of Showa University (permit number: 09074), which operates following the Japanese Government for the care and use of laboratory animals.

\section{LPS-induced ALI model establishment}

The mice intratracheally received $5 \mathrm{mg} / \mathrm{ml}$ LPS (Sigma-Aldrich, St. Louis, MO, USA) in $20 \mu \mathrm{l}(100 \mu \mathrm{g} /$ mouse) phosphate-buffered saline (PBS) on day 0 to induce a murine ALI model. Control mice received PBS. They were euthanized on day 1, 3, 5, or 7 . Furthermore, $5 \mu \mathrm{g} / \mathrm{ml}$ anti-IL-34 antibody (AF5195; R\&D Systems, Minneapolis, MN, USA) in $20 \mu \mathrm{l}$ (100 ng/mouse) PBS was intranasally administered to mice for three consecutive days before LPS stimulation. They were also euthanized on day 1, 3, 5 , or 7 (Fig. 1). PBS was administered to control mice.

Measurement of cells and cytokines levels in bronchoalveolar lavage fluid

The mice were euthanized on day $1,3,5$, or 7 , and the trachea was exteriorized via blunt dissection. Subsequently, a small-caliber needle was inserted and secured within the airway to instill normal saline for bronchoalveolar lavage fluid (BALF) collection. The airway was successively washed thrice with 350 $\mu \mathrm{l}$ of normal saline, which was instilled and gently aspirated to obtain BALF. Moreover, the BALF was centrifuged $\left(3,000 \mathrm{rpm}, 7 \mathrm{~min}, 4^{\circ} \mathrm{C}\right)$, and the cells were washed and resuspended in $100 \mu \mathrm{l}$ normal saline. The total cell count was calculated using a hemocytometer after staining with Turk's solution (Wako, Osaka, Japan). Differential cell count was conducted on cytospin cell preparations (Cytospin 3; Shandon, Pittsburgh, PA, USA) and stained with DiffQuick (Dade Behring Inc., Newark, DE, USA). A differential cell count was performed on 400 cells using the standard morphological criteria.

The IL-34 levels in BALF and cell culture supernatant 


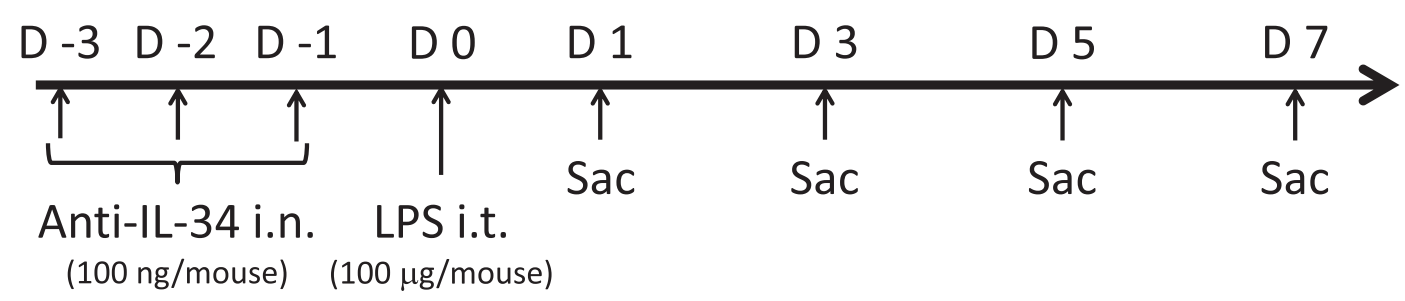

Fig. 1. Protocol for the murine model of LPS-induced ALI and treatment of anti-IL-34 antibody. LPS ; lipopolysaccharide, ALI ; acute lung injury, IL-34 ; interleukin-34, i.n. ; intranasally, i.t. ; intratracheally.

were measured using enzyme-linked immunosorbent assay (ELISA) kits (R\&D Systems) according to the manufacturer's instructions.

\section{Gene expression measurement in the lung tissue}

The lung tissue was homogenized in TRIzol reagent (Invitrogen, Carlsbad, CA, USA) and extracted with chloroform. RNA was precipitated with isopropanol, washed with $75 \%$ ethanol, and resuspended in RNasefree water. The total RNA was treated with DNase I (Promega, Madison, WI, USA). The SuperScript II first-strand synthesis system (Invitrogen) was used to synthesize cDNA using reverse transcription in the presence of oligo dT primers from $1 \mu \mathrm{g}$ of total RNA. The amplification of each cDNA stand was achieved with a Taq DNA polymerase (Promega). The cDNA expression was measured by quantitative polymerase chain reaction (qPCR) using the master mix of SYBR Green/ROX (Qiagen, Frederick, MD, USA) in the C1000 Touch thermal cycler (BIORAD, Hercules, CA, USA). The ratio of each mRNA molecule relative to the glyceraldehyde 3-phosphate dehydrogenase (GAPDH) mRNA was calculated with the ${ }^{\Delta \Delta} \mathrm{Ct}$ threshold cycle method. The mouse primers for IL-34, tumor necrosis factor- $\alpha$ (TNF- $\alpha$ ), CCL22, and arginase-1 (Arg1) were obtained from Qiagen.

\section{Lung histopathology analysis}

After obtaining BALF, the lungs were dissected from the chest and inflated with $500 \mu$ l of $10 \%$ formalin in PBS. The lung tissues were dehydrated in ethanol, embedded in paraffin, sectioned $(5 \mu \mathrm{m})$, and stained with Masson's trichrome. Histopathological examination of the lung was performed with an optical microscope under $\times 100$ magnification. The percentage of collagen deposition area in the entire lung field was measured in three lung sections per mouse by hybrid cell count using a digital microscope (BZ-X800; Keyence Tokyo, Japan) as previously described to evaluate pulmonary fibrosis ${ }^{21}$.

The lung sections mounted on amino alkylsilanecoated glass slides were deparaffinized and rehydrated.
The sections were heated with citrate buffer $(0.1 \mathrm{M}$, $\mathrm{pH}:$ 6) for $10 \mathrm{~min}$ in a microwave oven for antigen retrieval, followed by incubation in $0.3 \%$ hydrogen peroxide for $30 \mathrm{~min}$ to block endogen peroxidase. After repeated washing with PBS, protein blocking was performed with a serum-free protein block solution (X2w0909; DAKO, Carpinteria, CA, USA) for $15 \mathrm{~min}$. Monoclonal mouse anti-IL-34 antibodies (sc-517217; Santa Cruz Biotechnology, Santa Cruz, CA, USA) and its isotype control were used as the primary antibodies, along with broad-spectrum secondary antibodies (Envision Dual Link SystemHRP, K4063; DAKO). After flushing, the sections were treated with peroxidase-conjugated streptavidin (Thermo Fisher Scientific, Rockford, IL, USA), followed by treatment with 3,3'-diaminobenzidine (liquid DAB + substrate, K3467 ; DAKO) for antibody reaction visualization. The lung sections were counterstained using Mayer's hematoxylin and examined under light microscopy. Each airway from the four lung sections was examined per mouse using a $0-5+$ score classification to determine the IL-34 expression on the surface of the airway epithelium. For $0-5+$ scoring, the isotype control slide was set as 0 , and the darkest dyed slide as $5+$ (Fig 2A-E). The analysis was performed by two independent observers blinded to group information.

Investigating the IL-34 expression by LPS stimulation in vitro

BEAS-2B (a human airway epithelial cell line transformed with an adenovirus $12-\mathrm{SV} 40$ virus hybrid; American Type Culture Collection, Manassas, VA, USA) and THP-1 (a human peripheral blood monocyte cell line from acute monocytic leukemia; American Type Culture Collection) cells were cultured with $1 \mu \mathrm{g} / \mathrm{ml}$ of LPS in Dulbecco modified Eagle medium (Biofluids, Inc., Rockville, MD, USA) containing $10 \%$ fetal bovine serum, $100 \mathrm{U} /$ $\mathrm{ml}$ penicillin, and $100 \mathrm{ng} / \mathrm{ml}$ streptomycin (Fisher Scientific, Gaithersburg, MD, USA) at $37^{\circ} \mathrm{C}$ in humidified air containing $5 \% \mathrm{CO}_{2}$. The cell culture 
A. LPS D1

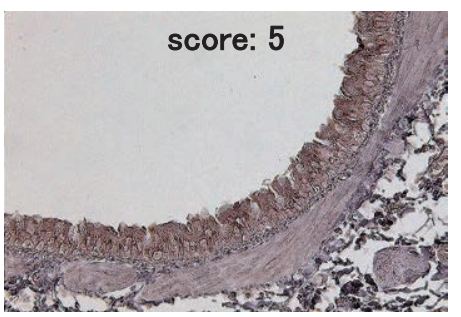

D. Ctl

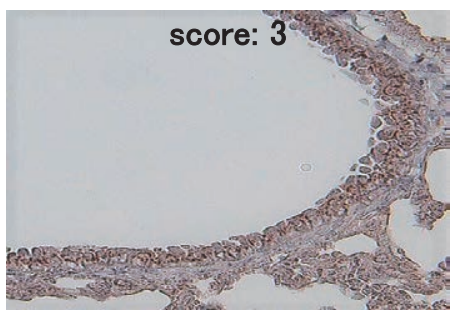

\section{B. LPS D3}

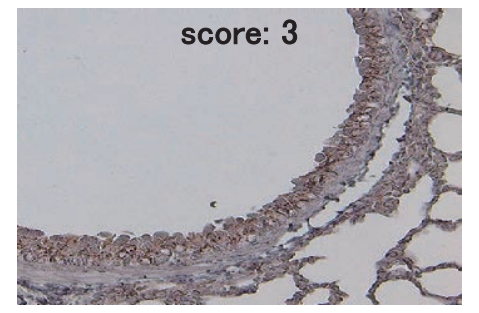

E. Isotype



\section{LPS D7}
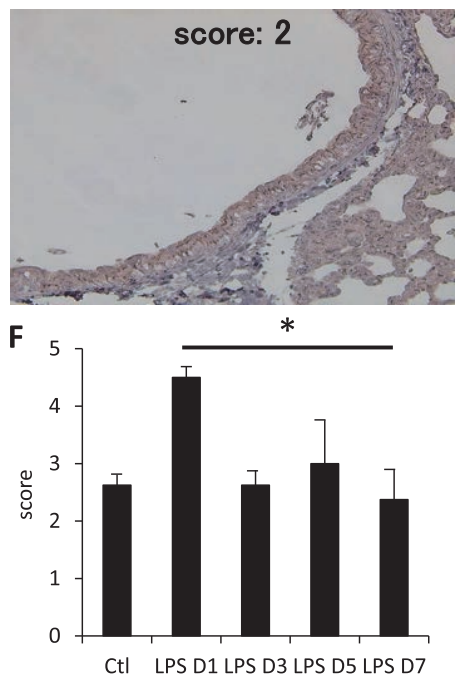

Fig. 2. Representative lung sections are showing immunohistochemistry staining for IL-34 (brown; A-E). Statistical analysis for IL-34 expression was examined using a 0-5+ scale classification ( $F)$. The results are expressed as mean $\pm \operatorname{SEM~(~} n=8$ for all groups). ${ }^{*} p<0.05$. IL-34 interleukin-34. isotype the antibody that has the same primary antibody and does not react with a specific protein.

supernatants were collected at 1, 3, 6, 12, and 24 $\mathrm{h}$ after LPS incubation. BEAS-2B cells were also cultured with $10 \mathrm{ng} / \mathrm{ml}$ of TNF- $\alpha$ for $24 \mathrm{~h}$, and the supernatants were collected. The IL-34 and TNF- $\alpha$ levels in the supernatants were measured using ELISA kits (BioSource International, Camarillo, CA, USA) according to the manufacturer's instructions. Moreover, qPCR was performed as previously described. The human primers for IL-34 and TNF- $\alpha$ were obtained from Qiagen.

\section{Statistical analysis}

Comparisons between the two groups were performed using unpaired Student's t-tests. A one-way analysis of variance with Sidak's correction for multiple comparisons was performed to compare three or more groups. Comparisons were performed with the $\mathrm{JMP}^{\circledR}$-software (SAS Institute Japan, Tokyo, Japan). Data are expressed as the mean \pm standard error of the mean (SEM). A p-value $<0.05$ was considered significant. All experiments were performed at least twice with four animals per group.

\section{Results}

\section{LPS treatment induced airway inflammation}

First, the progressive induction of lung inflammation caused by LPS over time was investigated. A significant increase was observed in the total cell count in the LPS-treated mice and the neutrophil count in the BALF on day 1 compared with that in the control mice $(49,375 \pm 1,219$ vs. $941,250 \pm 68,859$, $\mathrm{p}<0.01, \mathrm{n}=8$ for each; Fig. 3A, B). The expansion of these cells continued until day 3. On day 7, the percentage of neutrophils decreased compared with that on day 1 , while the percentage of macrophages increased $(7.67 \pm 1.45$ vs $90.6 \pm 1.75, \mathrm{p}<0.01,80.67 \pm 1.33$ vs. $7.00 \pm 1.52, p<0.01, n=5$ and 6 , respectively; Fig. 3B).

\section{LPS treatment induced IL-34 expression}

The IL-34 expression in BALF and lung tissue was measured to ascertain the role of IL-34. The expression and production of IL-34 significantly increased in the lung tissue and BALF following LPS treatment on day 1 and returned to baseline levels after day 3 (Fig. 3D, E). The IL-34 distribution in the lung was also investigated using immunohistochemistry staining. IL-34 was mainly detected on the surface of the epithelial cells. IL-34 expression was significantly higher in LPS-treated mice on day 1 than in the control mice $(\mathrm{n}=8$ for each group; Fig. $2 \mathrm{~F})$.

\section{Anti-IL-34 antibody inhibits the LPS-induced airway inflammation and pulmonary fibrosis}

The mice with an anti-IL-34 antibody were treated next to investigate the role of IL-34 in the LPSinduced lung injury mouse model. A significant reduction was observed in the total cell count in BALF on day 3 after treatment with anti-IL-34 antibodies $(1,193,750 \pm 123,191$ vs. $746,563 \pm 58,604$, $\mathrm{p}=0.006, \mathrm{n}=8$ for each group ; Fig. 4A). Moreover, the number of macrophages in BALF significantly 
A. Total cells

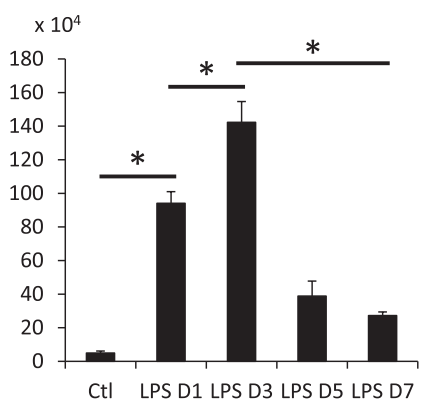

B. Differential cell percentages

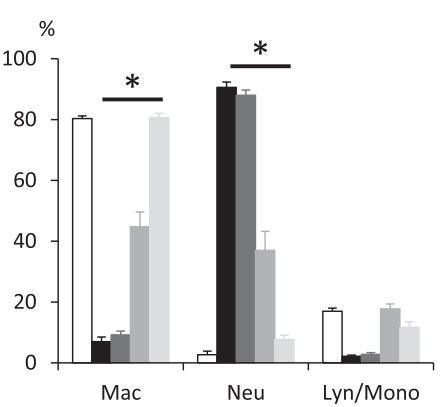

C. Differential cell counts

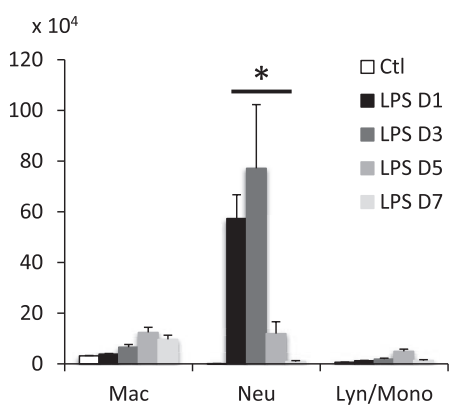

D. IL-34-mRNA expression in lung tissue

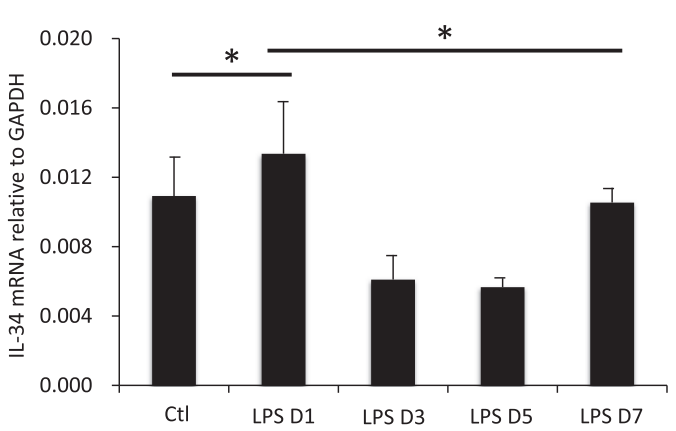

E. IL-34 concentration in BALF

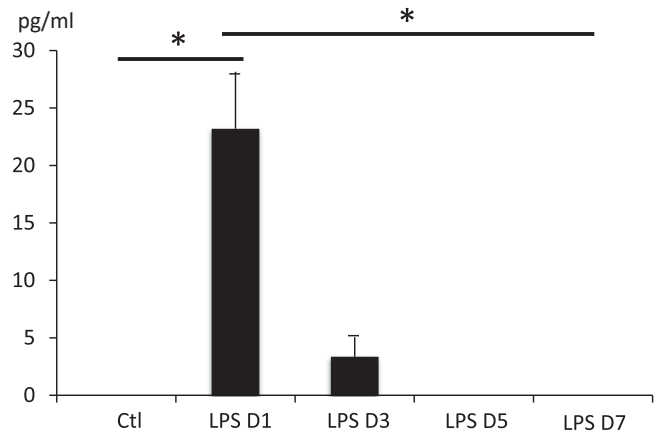

Fig. 3. Analysis of inflammatory cells and cytokines in the murine model of LPS-induced ALI. The total number of inflammatory cells $(A)$ and differential cell percentages and cell counts of the inflammatory cells (B and C) in BALF. Analysis of IL-34 gene expression in the lung tissue by qPCR (D). Analysis of cytokine production in the BALF by ELISA (E). The results are expressed as mean \pm SEM $(n=5-8) .{ }^{*} p<0.05$. LPS lipopolysaccharide, ALI acute lung injury, BALF bronchoalveolar lavage fluid, IL-34 interleukin-34, qPCR quantitative polymerase chain reaction, ELISA enzyme-linked immunosorbent assay.

decreased from days 1 to 7 (Fig. 4B). The examination of lung tissue homogenate revealed that the CCL22 expression was inhibited from days 1 to 7 and that of Arg1 was inhibited from days 3 to 7 after treatment with anti-IL-34 antibodies (Fig. 4C, D). Pulmonary fibrosis evaluation using histological analysis revealed a reduction in pulmonary fibrosis on day 5 in the anti-IL-34 antibody-treated mice (exposed to LPS) compared with that in the LPS-exposed untreated mice (percentage of collagen deposition, $0.22 \pm 0.04$ vs. $0.40 \pm$ $0.07, \mathrm{p}=0.03, \mathrm{n}=5$ for each group; Fig. 5).

\section{TNF- $\alpha$ stimulation induced IL-34 production in vitro}

Finally, BEAS-2B and THP-1 cells were stimulated with LPS in vitro to determine IL-34 source production. Neither the BEAS-2B cells nor the THP1 cells were found to induce the IL-34 production upon stimulation with LPS (Fig. 6). However, there was significant induction of TNF- $\alpha$ gene expression and production in the THP-1 cells exposed to LPS for $3 \mathrm{~h}$, which reduced after $24 \mathrm{~h}$ of LPS stimulation (Fig. 7A, B). IL-34 production in the BEAS-2B cells stimulated with TNF- $\alpha$ for $24 \mathrm{~h}$ was significantly higher than that in the unstimulated cells (Fig. 7C).

\section{Discussion}

The present study investigated the role of IL-34 in a murine model of LPS-induced lung injury by focusing on airway inflammation and pulmonary fibrosis.

The status of the LPS-induced lung injury model was examined over days $1,3,5$, and 7 , before examining the role of IL-34 in ALI. The number of total cells in BALF started increasing on day 1, peaked on day 3 , and subsequently decreased on day 5. The number of neutrophils increased with the increase in the total cell count whereas the number of macrophages increased as the total cell count decreased. Fujino et al. reported that LPS-treated animals undergo resolution of lung inflammation and fibrosis 7 days after LPS exposure ${ }^{22}$. Another study has also demonstrated the involvement of macrophages in pulmonary fibrosis ${ }^{23}$, and the results of the current study are consistent with those of previous studies. These results suggest that 


\section{A. Total cells}

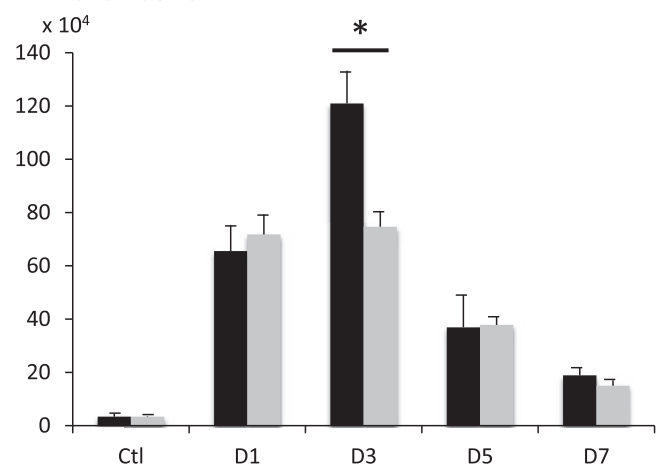

C. CCL22-mRNA expression in lung tissue

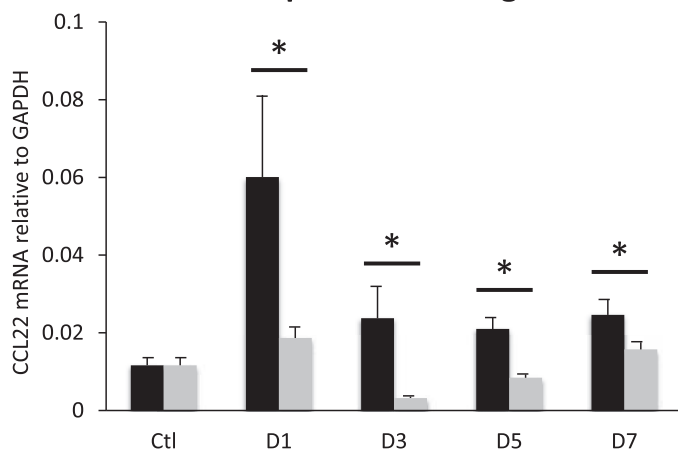

B. Number of macrophages



D. Arg1-mRNA expression in lung tissue

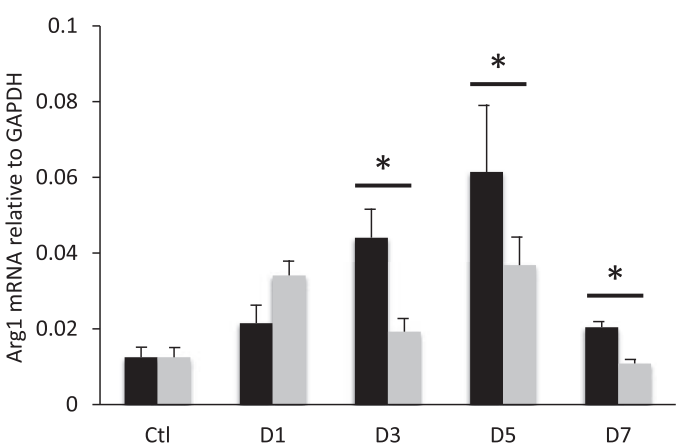

Fig. 4. Analysis of inflammatory cells and gene expression of $M 2$ macrophage markers in the murine model of LPS-induced ALI treated with anti-IL-34 antibody. The total number of inflammatory cells (A) and the number of macrophages (B) in the BALF. Analysis of gene expression of CCL22 and Arg1 in the lung tissue by qPCR ( $C$ and $D)$. The results are expressed as mean \pm SEM $(n=8-12)$. ${ }^{*} p<0.05$. LPS lipopolysaccharide, ALI acute lung injury, IL-34 interleukin-34, BALF bronchoalveolar lavage fluid, Arg1 arginase-1, qPCR quantitative polymerase chain reaction.

\section{A. LPS D5}
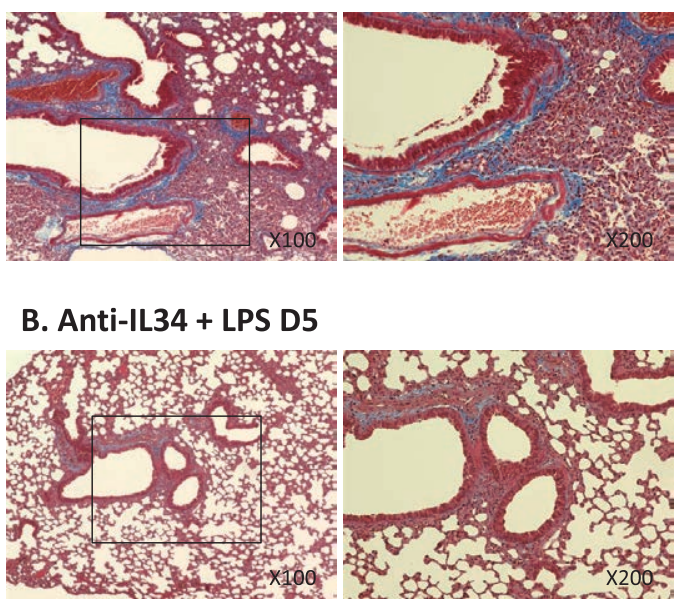

\section{Percentages of collagen deposition area}

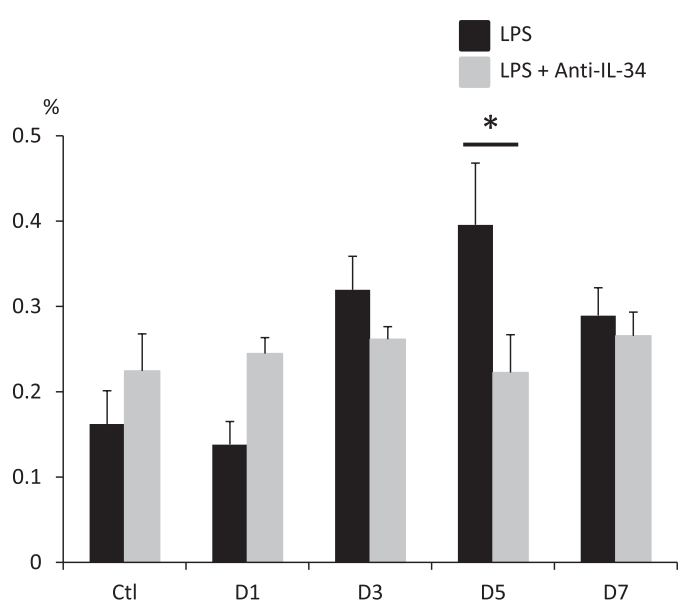

Fig. 5. Representative lung sections are showing Masson's trichrome staining for analysis of lung fibrosis ( $A$ and $B$ ). The percentage of collagen deposition area in the entire lung field was measured (B). The results are expressed as mean \pm SEM $(n=4-5) .{ }^{*} p<0.05$. LPS lipopolysaccharide, IL-34 interleukin-34.

neutrophilic inflammation occurs in the lung at the early stage of LPS exposure, which is replaced by macrophage proliferation, which may in turn promote pulmonary fibrosis.
The role of IL-34 was investigated first by examining the IL-34 expression in BALF and lung tissue of the LPS-induced murine model of lung injury. A significant increase in IL-34 expression was 
A. IL-34-mRNA expression in BEAS-2B cells under LPS stimulation

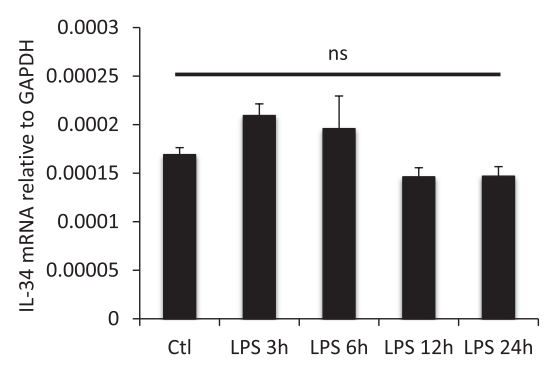

C. IL-34-mRNA expression in THP-1 cells under LPS stimulation

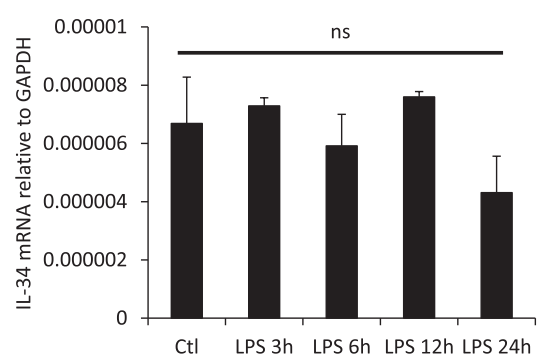

B. IL-34 concentration in the culture media of BEAS-2B cells under LPS stimulation

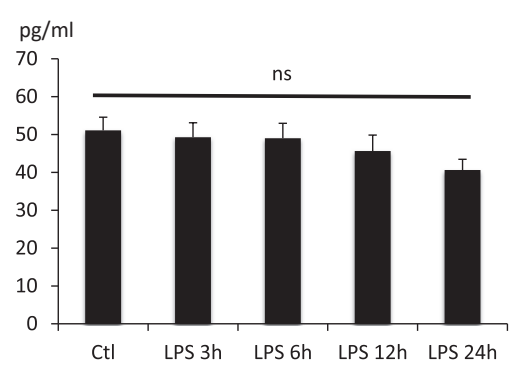

D. IL-34 concentration in the culture media of THP-1 cells under LPS stimulation

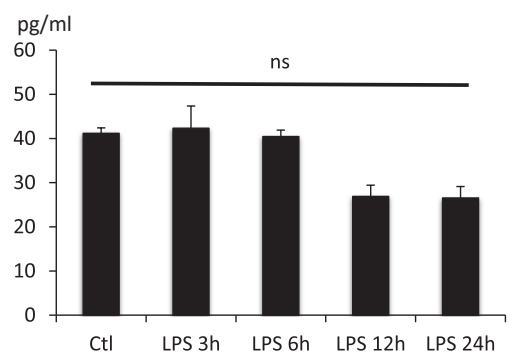

Fig. 6. BEAS-2B cells and THP-1 cells were stimulated with LPS for $3,6,12$, and $24 \mathrm{~h}$. Analysis of IL-34 gene expression in BEAS-2B cells stimulated with LPS (A). Analysis of IL-34 production in the supernatant after stimulation of BEAS-2B cells with LPS (B). Analysis of IL-34 gene expression in THP-1 cells stimulated with LPS (C). Analysis of IL-34 production in the supernatant after stimulation of THP-1 cells with LPS (D). The results are expressed as mean \pm SEM $(n=6$ for all groups). LPS lipopolysaccharide, IL-34 interleukin-34, ns not significant.

A. TNF- $\alpha-m R N A$ expression in THP-1 cells under LPS stimulation

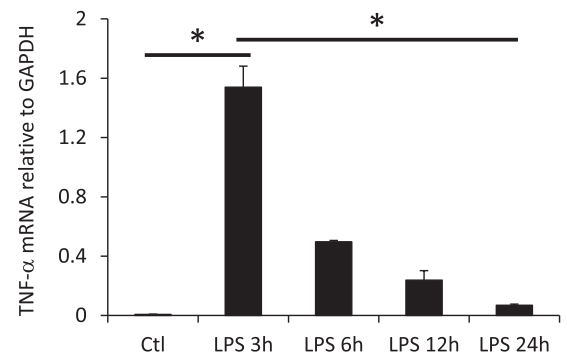

C. IL-34 concentration in the culture media of BEAS-2B cells under TNF- $\alpha$ stimulation

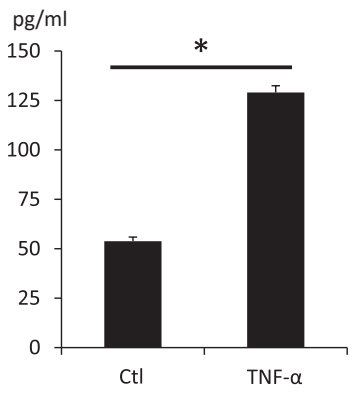

B. TNF- $\alpha$ concentration in the culture media of THP-1 cells under LPS stimulation

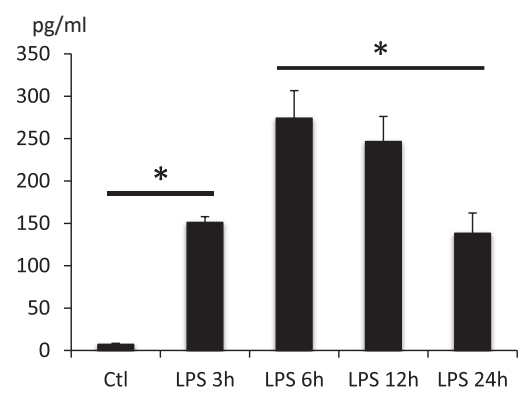

Fig. 7. Analysis of TNF- $a$ gene expression in THP-1 cells stimulated with LPS for $3,6,12$, and $24 \mathrm{~h}$ (A). Analysis of TNF- $a$ production in the supernatant after stimulation of THP-1 cells with LPS for $3,6,12$, and $24 \mathrm{~h}$ (B). Analysis of production of IL-34 in the supernatant after stimulation of BEAS-2B cells with TNF- $a$ (C). The results are expressed as mean \pm SEM ( $n=6$ for all groups). ${ }^{*} p<0.05$. TNF- $a$ tumor necrosis factor- $a$, LPS lipopolysaccharide, IL-34 interleukin-34. 
observed on day 1 in both samples. Furthermore, IL-34 was confirmed to be mainly expressed in the airway epithelium, which peaked on day 1 . Thus, IL-34 was inferred IL-34 to be mainly produced by the airway epithelium during the early stage of LPS-induced injury and may contribute to the pathogenesis of lung injury. Previous studies reported that IL-34 is involved in the fibrosis of organs such as the kidney and liver ${ }^{24,25}$. However, no study has reported on the IL-34 involvement in pulmonary inflammation and lung injury. The present study was the first to confirm the main expression site and production chronology of IL-34 in the LPS-induced lung injury model.

Further investigations were conducted by administering anti-IL-34 antibodies to the LPS-induced lung injury model. The anti-IL-34 antibodies reduced the number of total cells in BALF on day 3 and the number of macrophages from days 1 to 7 , showing that the IL-34 blockade inhibits the inflammation caused by LPS stimulation. Moreover, the expression of CCL22 and Arg1, markers for M2 macrophages ${ }^{26}$, was decreased by IL-34 blockade. Preisser et al. reported that IL-34 is involved in the differentiation of profibrotic macrophages in the liver ${ }^{27}$. However, its role in lung macrophages is unknown. The present study suggests that IL-34 may play a role in inducing the differentiation of immature macrophages into anti-inflammatory macrophages. Moreover, in this study, CCL22 and Arg1 were examined for gene expression in the whole lung and were expressed in cells other than macrophages. Therefore, further studies specific to macrophages are needed. Moreover, histological analysis revealed that pulmonary fibrosis was inhibited by blockade of IL-34 on day 7. Overall, this suggests that IL-34 induces pulmonary inflammation and lung injury, resulting in pulmonary fibrosis induction.

The mechanism of IL-34 production in the airway epithelium and its effect on the differentiation of macrophages using in vitro examination of BEAS2B and THP-1 cells was elucidated. IL-34 production did not increase over time and was not significantly different from any other group when cell lines were exposed to LPS. This means that both the BEAS2B and THP-1 cells are unlikely to produce IL-34 in response to LPS stimulation alone. Thus, other factors may be necessary for IL-34 production. LPS increased TNF- $\alpha$ production in the THP- 1 cells, and IL-34 production was induced by TNF- $\alpha$ in BEAS$2 \mathrm{~B}$ cells. The results of the current study suggest that TNF- $\alpha$ is produced by LPS-stimulated monocytes or macrophages, and the airway epithelium produces IL-34 in response to stimulation by TNF- $\alpha$.

A limitation of this study was that the murine ALI model was created using only LPS since other factors also contribute to ALI in real-world settings. Furthermore, the mechanisms of IL-34 production in the airway epithelium are still unknown.

In conclusion, the present study suggested that TNF- $\alpha$ produced by LPS stimulation induces the production of IL-34 in the airway epithelium. It also showed that IL-34 induces pulmonary inflammation and causes ALI.

\section{Acknowledgments}

The authors thank Manami Matsuda, Teru Haba, and Kyoko Inui for their skillful technical assistance.

\section{Conflict of interest disclosure}

The authors have no conflict of interest to declare.

\section{References}

1. Wei $S$, Nandi $S$, Chitu V, et al. Functional overlap but differential expression of CSF-1 and IL-34 in their CSF-1 receptor-mediated regulation of myeloid cells. $J$ Leukoc Biol. 2010;88:495-505.

2. Wang $\mathrm{Y}$, Szretter KJ, Vermi $\mathrm{W}$, et al. IL-34 is a tissue-restricted ligand of CSF1R required for the development of Langerhans cells and microglia. Nat Immunol. 2014;13:753-760.

3. Droin N, Solary E. Editorial: CSF1R, CSF-1, and IL-34, a "menage a trois" conserved across vertebrates. J Leukoc Biol. 2010;87:745-747.

4. Lin $\mathrm{H}$, Lee $\mathrm{E}$, Hestir $\mathrm{K}$, et al. Discovery of a cytokine and its receptor by functional screening of the extracellular proteome. Science. 2008;320:807-811.

5. Eda $\mathrm{H}$, Zhang $\mathrm{J}$, Keith $\mathrm{RH}$, et al. Macrophage-colony stimulating factor and interleukin-34 induce chemokines in human whole blood. Cytokine. 2010;52:215-220.

6. Felix J, De Munck S, Verstraete $\mathrm{K}$, et al. Structure and assembly mechanism of the signaling complex mediated by human CSF-1. Structure. 2015;23:1621-1631.

7. Stanley ER, Chitu V. CSF-1 receptor signaling in myeloid cells. Cold Spring Harb Perspect Biol. 2014;6:a021857. (accessed 2021 Feb 20) Available from: https://www.ncbi.nlm.nih.gov/pmc/articles/ PMC4031967/pdf/cshperspect-RTK-a021857.pdf

8. Bostrom EA, Lundberg P. The newly discovered cytokine IL-34 is expressed in gingival fibroblasts, shows enhanced expression by pro-inflammatory cytokines, and stimulates osteoclast differentiation. PLOS One. 2013;8:e81665. (accessed 2021 Feb 20) Available from: https ://www.ncbi.nlm.nih.gov/pmc/articles/ PMC3858262/pdf/pone.0081665.pdf 
9. Wang Y, Colonna M. Interkeukin-34, a cytokine crucial for the differentiation and maintenance of tissue resident macrophages and Langerhans cells. Eur $J$ Immunol. 2014;44:1575-1581.

10. Ciccia F, Alessandro R, Rodolico V, et al. IL-34 is overexpressed in the inflamed salivary glands of patients with Sjogren's syndrome and is associated with the local expansion of pro-inflammatory CD14 (bright) CD16+ monocytes. Rheumatology (Oxford). 2013;52:1009-1017.

11. Moon SJ, Hong YS, Ju JH, et al. Increased levels of interleukin 34 in serum and synovial fluid are associated with rheumatoid factor and anticyclic citrullinated peptide antibody titers in patients with rheumatoid arthritis. J Rheumatol. 2013;40:1842-1849.

12. Franze E, Marafini I, De Simone V, et al. Interleukin-34 induces cc-chemokine ligand 20 in gut epithelial cells. J Crohns Colitis. 2016;10:87-94.

13. Bhatia M, Zemans RL, Jeyaseelan S. Role of chemokines in the pathogenesis of acute lung injury. $A m \mathrm{~J}$ Respir Cell Mol Biol. 2012;46:566-572.

14. Ashbaugh DG, Bigelow DB, Petty TL, et al. Acute respiratory distress in adults. Lancet. 1967;2:319-323.

15. Faist $\mathrm{E}$, Baue $\mathrm{AE}$, Dittmer $\mathrm{H}$, et al. Multiple organ failure in polytrauma patients. J Trauma. 1983;23:775787.

16. MacLaren R, Jung R. Stress-dose corticosteroid therapy for sepsis and acute lung injury or acute respiratory distress syndrome in critically ill adults. Pharmacotherapy. 2002;22:1140-1156.

17. Maldonado RF, Sa-Correia I, Valvano MA. Lipopolysaccharide modification in Gram-negative bacteria during chronic infection. FEMS Microbiol Rev. 2016;40: 480-493.

18. Ginhoux F, Jung S. Monocytes and macrophages: developmental pathways and tissue homeostasis. Nat Rev Immunol. 2014;14:392-404.
19. Jakubzick CV, Randolph GJ, Henson PM. Monocyte differentiation and antigen-presenting functions. Nat Rev Immunol. 2017;17:349-362.

20. Sordet O, Rebe C, Plenchette S, et al. Specific involvement of caspases in the differentiation of monocytes into macrophages. Blood. 2002;100:4446-4453.

21. Miyata Y, Ohta S, Tanaka A, et al. The effect of bronchoconstriction by methacholine inhalation in a murine model of asthma. Int Arch Allergy Immunol. 2020;181:897-907.

22. Fujino $\mathrm{N}$, Kubo $\mathrm{H}$, Suzuki $\mathrm{T}$, et al. Administration of a specific inhibitor of neutrophil elastase attenuates pulmonary fibrosis after acute lung injury in mice. Exp Lung Res. 2012;38:28-36.

23. Laskin DL, Malaviya R, Laskin JD. Role of macrophages in acute lung injury and chronic fibrosis induced by pulmonary toxicants. Toxicol sci. 2019;168: 287-301.

24. Baek JH, Zeng R, Weinmann-Menke J, et al. IL-34 mediates acute kidney injury and worsens subsequent chronic kidney disease. J Clin Invest. 2015;125:31983214.

25. Wang $Y Q$, Cao WJ, Gao YF, et al. Serum interleukin-34 level can be an indicator of liver fibrosis in patients with chronic hepatitis B virus infection. World J Gastroenterol. 2018;24:1312-1320.

26. Saba TG, Chung Y, Hong JY, et al. Rhinovirusinduced macrophage cytokine expression does not require endocytosis or replication. Am J Respir Cell Mol Biol. 2014;50:974-984.

27. Preisser L, Miot C, Le Guillou-Guillemette $\mathrm{H}$, et al. IL-34 and macrophage colony-stimulating factor are overexpressed in hepatitis $\mathrm{C}$ virus fibrosis and induce profibrotic macrophages that promote collagen synthesis by heaptic stellate cells. Hepatology. 2014;60: 1879-1890. 